\title{
Measurement of core velocity fluctuations and the dynamo in a reversed-field pinch
}

D. J. Den Hartog ${ }^{1}$, J. T. Chapman², D. Craig, G. Fiksel, P. W. Fontana, S. C. Prager, and J. S. Sarff

Department of Physics, University of Wisconsin-Madison, 1150 University Avenue, Madison, WI 53706

Plasma flow velocity fluctuations have been directly measured in the high temperature magnetically confined plasma in the Madison Symmetric Torus (MST) Reversed-Field Pinch (RFP) [R. N. Dexter et al., Fusion Tech. 19, 131 (1991)]. These measurements show that the flow velocity fluctuations are correlated with magnetic field fluctuations such that the electromotive force $\langle\tilde{\mathbf{v}} \times \tilde{\mathbf{B}}\rangle$ approximately balances parallel Ohm's law, $E_{\|}+\langle\tilde{\mathbf{v}} \times \tilde{\mathbf{B}}\rangle_{\|}=\eta J_{\|}$. This initial measurement is subject to limitations of spatial localization and other uncertainties, but is evidence for sustainment of the RFP magnetic field configuration by the magnetohydrodynamic (MHD) dynamo, $\langle\tilde{\mathbf{v}} \times \tilde{\mathbf{B}}\rangle$. Both the flow velocity and magnetic field fluctuations are the result of global resistive MHD modes of helicity $m=1, n=5-10$ in the core of MST. Chord-averaged flow velocity fluctuations are measured in the core of MST by recording the Doppler shift of impurity line emission with a specialized high resolution and throughput grating spectrometer. Magnetic field fluctuations are recorded with a large array of small edge pickup coils, which allows spectral decomposition into discrete modes and subsequent correlation with the velocity fluctuation data. PACS: $52.30 .-\mathrm{q}, 52.25 . \mathrm{Gj}, 52.30 . \mathrm{Jb}, 52.70 . \mathrm{Kz}$

1djdenhar@facstaff.wisc.edu

2Siemens Medical Systems, Inc., Nuclear Medicine Group, 2501 North Barrington Road, Hoffman Estates, IL 60195 


\section{INTRODUCTION}

In the Madison Symmetric Torus ${ }^{1}$ (MST) reversed-field pinch (RFP), we have measured the plasma flow velocity fluctuations $\tilde{\mathbf{v}}$ with a fast, high-resolution spectrometer, the magnetic field fluctuations $\tilde{\mathbf{B}}$ with an edge coil array and insertable probe, and correlated the two quantities to show that $\langle\tilde{\mathbf{v}} \times \tilde{\mathbf{B}}\rangle_{\|}$approximately balances parallel Ohm's law, $E_{\|}+\langle\tilde{\mathbf{v}} \times \tilde{\mathbf{B}}\rangle_{\|}=\eta J_{\|}$. Subject to experimental limitations, this is direct experimental evidence that the magnetohydrodynamic (MHD) dynamo term, $\langle\tilde{\mathbf{v}} \times \tilde{\mathbf{B}}\rangle$, is the dominant emf sustaining the RFP magnetic field configuration against resistive decay in MST.

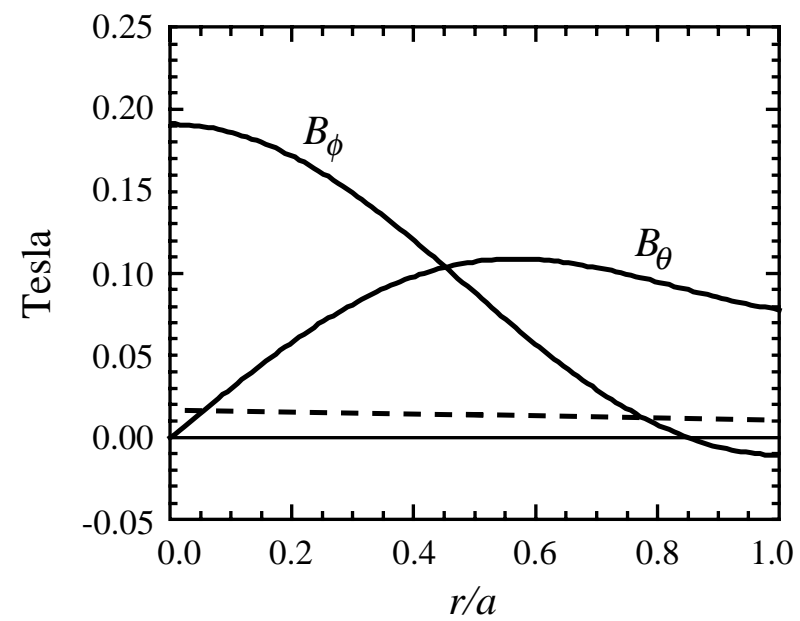

Fig. 1. The solid lines illustrate the magnetic field configuration of an RFP plasma in MST. The dashed line is the toroidal field pre-fill prior to formation of the RFP plasma.

\section{A. RFP equilibrium}

Like the tokamak, the RFP is a toroidal magnetic field configuration capable of confining a high temperature plasma. However, unlike a tokamak where the toroidal magnetic field $B_{\phi}$ is

much greater than the poloidal magnetic field $B_{\theta}$, in an RFP $B_{\phi} \approx B_{\theta}$. Furthermore, in an RFP the toroidal magnetic field actually goes to zero and then reverses sign near the edge of the plasma (Fig. 1). This magnetic field configuration is produced and sustained by conversion of poloidal flux to toroidal flux inside of a conducting shell, a process often referred to as the "RFP dynamo." 
The RFP attains its unique magnetic field configuration via a relaxation process in which magnetic helicity is roughly conserved while magnetic energy is minimized. ${ }^{2,3}$ The resulting magnetic field configuration is described by $\nabla \times \mathbf{B}=\lambda \mathbf{B}$, where $\lambda$ is a constant. Solving for the equilibrium magnetic field configuration consistent with this equation in a periodic cylinder yields the Bessel Function Model (BFM): $B_{\phi}=B_{0} J_{0}(\lambda r)$ and $B_{\theta}=B_{0} J_{1}(\lambda r)$, where $J_{0}$ and $J_{1}$ are first and second order Bessel functions and $\hat{\phi}=\hat{z}$ for a cylinder. The BFM predicts that when sufficient volt-seconds are applied to the plasma, $\lambda$ will increase. $J_{0}$ then swings through its first zero at $r<$ $a$ such that $B_{\phi}$ reverses direction in the plasma, a signature feature of RFP equilibrium. Thus the RFP is a "spontaneous" configuration, arising from the "relaxation" of the plasma into a minimum energy state. The exact mechanism for the relaxation is not addressed in this simple theory.

Because the equilibria obtained in experiment are not accurately described by the BFM, they are not fully relaxed into a minimum energy state. Nevertheless, we refer to field profiles which approach a constant $\lambda(r)$ as "relaxed" and describe the relative flattening of $\lambda(r)$ as a relaxation event.

\section{B. Ohm's law imbalance in the RFP}

As mentioned in the previous section, current is driven in an RFP plasma by an externally applied $E_{\phi}$ produced by a transformer. In the core this emf aligns with the magnetic field, producing $J_{\phi}$ and generating the observed $B_{\theta}$. However, near the edge of the plasma $\mathbf{B}$ is predominantly poloidal while the small $B_{\phi}$ component goes through zero and reverses sign. The applied $E_{\phi}$ is oblique to this equilibrium $\mathbf{B}$ at the edge and thus cannot drive the edge current necessary to generate the $B_{\phi}$ observed in the core. If the externally applied $E_{\phi}$ were the only emf in 
the plasma, the resistive dissipation of poloidal current in the edge would be balanced only by the decay of toroidal flux, implying that the RFP configuration would decay away in a resistive diffusion time. This is not observed in experiment; rather the toroidal flux configuration is maintained as long as the external $E_{\phi}$ is applied by the transformer.

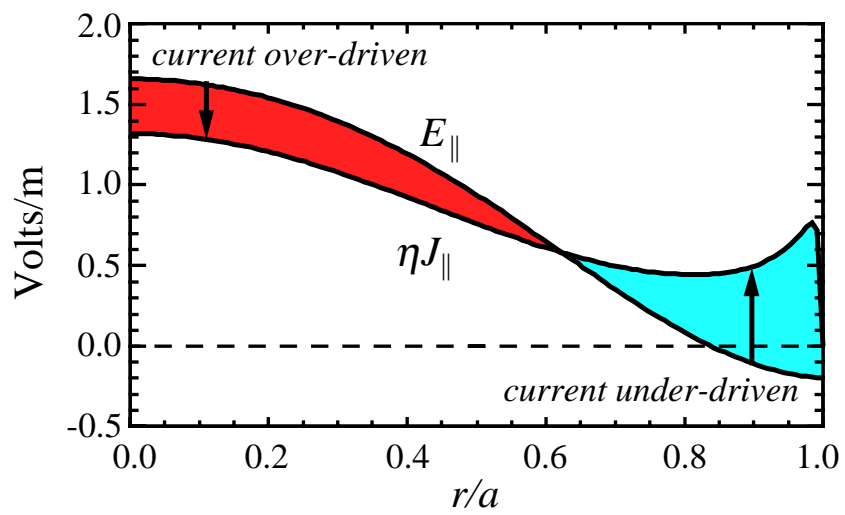

Fig. 2. An illustration showing that $\langle E\rangle_{\|} \neq \eta\langle J\rangle_{\|}$for an equilibrium modeled using typical MST parameters.

The imbalance in Ohm's law over the plasma radius is quantified in Fig. 2 where the applied $\langle E\rangle_{\|}$and observed $\eta\langle J\rangle_{\|}$are plotted (the " \langle\rangle " indicates an average over a flux surface).

In the edge the applied parallel electric field is too small or in the wrong direction to account for the driven current. In the core the applied field over-drives the necessary current, acting to peak the current profile and push the equilibrium away from the constant $\lambda$ relaxed state. Clearly, $\langle E\rangle_{\|} \neq \eta\langle J\rangle_{\|}$, we must postulate $\langle E\rangle_{\|}+\left\langle E_{d}\right\rangle_{\|}=\eta\langle J\rangle_{\|}$, where $E_{d}$ is often called the "dynamo electric field." In this paper we will present clear evidence that this field is equivalent to the dynamo term contained in single-fluid $\mathrm{MHD},\langle\tilde{\mathbf{v}} \times \tilde{\mathbf{B}}\rangle$, where correlated fluctuations in plasma flow velocity and magnetic field produce a non-zero average emf on a flux surface.

C. Diagnostic methods

Since the eigenfunctions of the resistive tearing mode magnetic fluctuations that are 
observed in all RFP's exhibit substantial amplitude at the edge of the plasma, measurement of the magnetic fluctuations in MST is done with toroidally and poloidally distributed arrays of magnetic pickup coils at the plasma edge. The plasma and the tearing modes rotate toroidally in the lab frame, so by analog integration of the signals from these coil arrays we are able to resolve the dominant spatial Fourier modes of the magnetic fluctuations. The plasma flow velocity fluctuations are much more difficult to measure. Their amplitude is a few percent of the thermal velocity of the plasma ions, meaning that typical measured $|\tilde{v}| \approx 1 \mathrm{~km} / \mathrm{s}$ in MST. Fortunately, these fluctuations are long wavelength, on the order of the minor radius $a$. Thus the spatial resolution available with passive emission Doppler spectroscopy is sufficient to measure the character and approximate amplitude of the velocity fluctuations. The Doppler spectrometer will be briefly described in Sec. III below, followed by a description of the data analysis techniques employed to quantify $\tilde{v}$ and the correlated product $\langle\tilde{\mathbf{v}} \times \tilde{\mathbf{B}}\rangle$. Measurement results in MST and a comparison to Ohm's law will also be presented in Sec. III. But first, in Sec. II, we present a brief history of work on the RFP dynamo and present a more detailed description of the MHD dynamo, concentrating on the nature of the fluctuations. Finally, Sec. IV is a summary and presents some implications of this work.

\section{THE RFP DYNAMO}

\section{A. Previous work}

In 1968 the first paper discussing in detail the properties of the "stable period" in the ZETA RFP was presented. ${ }^{4}$ This work showed that when the reversed-field configuration appeared, confinement increased and fluctuations decreased. However, the inherent nonlinearity of the RFP dynamo made the details of the process analytically intractable. Substantial progress began to be made in 1977 with the first observation of robust toroidal field reversal in a numerical simulation of the resistive MHD equations in a pinch configuration. ${ }^{5}$ The basic mechanism of field reversal and sustainment in the RFP was identified as the nonlinear evolution of $m=1$ kink instabilities in the 
presence of an externally applied toroidal voltage. Rapid progress in simulation since 1977 has resulted in 3-dimensional, well-resolved numerical solutions of resistive MHD equations that clearly illustrate the importance of the MHD dynamo in balancing Ohm's law in the RFP. ${ }^{6}$

Prior to the work described in this paper, experimental measurement of the RFP dynamo has been limited employment of a Langmuir probe and pickup coils to measure $\tilde{\mathbf{E}}_{\perp}, \nabla_{\perp} \tilde{P}_{e}$, and $\tilde{\mathbf{B}}$ in the edge plasma of several RFP's. ${ }^{7}$ By calculating $\left\langle\tilde{\mathbf{E}}_{\perp} \cdot \tilde{\mathbf{B}}_{\perp}\right\rangle$ (the fluctuating $\mathbf{E} \times \mathbf{B}$ drift dynamo term) and $\left\langle\nabla_{\perp} \tilde{P}_{e} \cdot \tilde{\mathbf{B}}_{\perp}\right\rangle$ (the fluctuating diamagnetic drift dynamo term) over an ensemble of discrete dynamo events, the balance of parallel Ohm's law was demonstrated. In relatively collisionless plasmas like those obtained in $\operatorname{MST}$ the $\left\langle\tilde{\mathbf{E}}_{\perp} \cdot \tilde{\mathbf{B}}_{\perp}\right\rangle$ term dominated the dynamo contribution. Unexpectedly, in the more collisional plasma in the edge of the TPE-1RM20 RFP, the diamagnetic term dominated. Thus, although the single-fluid MHD dynamo term appears to be the dominant RFP dynamo mechanism in simulation and the MST experiment, it may not apply in such a singular fashion to all RFP experiments. In particular, further two-fluid MHD simulation should be done to determine if other terms in Ohm's law become important in certain operational regimes.

In contrast to the MHD description of the RFP dynamo, a mechanism known as the "kinetic dynamo" has been proposed to explain the flattening of the current profile observed in the RFP. $^{8}$ In this theory, current generation arises from current flowing from the center to the edge along a stochastic magnetic field. Parallel electron momentum driven by the applied electric field in the core streams along the magnetic field to the edge region where the field is predominantly poloidal, thus flattening the parallel current profile. Recent Fokker-Planck simulation of electron distributions in the RFP with a parallel momentum diffusion set to be consistent with RechesterRosenbluth diffusivity suggest that the kinetic dynamo could produce the observed RFP equilibrium, ${ }^{9}$ although self-consistency constraints may limit the effectiveness of this 
mechanism. ${ }^{10}$ Experimental measurements of the electron momentum distribution at the edge of MST do not appear to be consistent with the streaming of fast electrons from the core to the edge. ${ }^{11}$ While the measurements described below provide strong evidence for MHD dynamo activity in the core and edge of MST, detailed commentary on the validity of the kinetic dynamo is outside the scope of this work.

Finally, we briefly comment on the relationship of the classical astrophysical dynamo to the RFP dynamo. An obvious difference is that in space the magnetic field is totally driven by the dynamo from a seed magnetic field, with no "applied" electric field. In the RFP only part of the field is self-generated. Also, while the RFP dynamo can be generally described in terms of an $\alpha$ effect, ${ }^{12}$ the velocity field cannot be taken to be given a priori and fixed for all time, as in some theories of the astrophysical dynamo. In the RFP, the magnetic field energy greatly exceeds the kinetic energy of the velocity fluctuations. This means that the backreaction of the magnetic field on the evolution of the velocity field cannot be ignored, even as an approximation. The equation of motion must be solved simultaneously with the induction equation and Ohm's law, making the RFP dynamo inherently nonlinear.

\section{B. Dynamo fluctuations in MST}

Fluctuation dynamics in the RFP are dominated by long wavelength normal modes that exhibit both resistive kink and resistive tearing characteristics. The nonlinear dynamics of these modes are described in detail in Ref. 6, so only a brief overview will be presented here. In MST, as in all other RFP's, low- $n, m=1$ magnetic modes resonant in the core dominate the magnetic fluctuation wavenumber spectrum. Resonance occurs when the safety factor, given approximately by $q(r) \approx r B_{\phi} / R B_{\theta}$, passes through a magnetic surface satisfying $q\left(r_{m n}\right)=m / n$, where $m$ is the poloidal mode number and $n$ is the toroidal mode number (Fig. 3). At this resonant surface, radial magnetic fluctuations produce magnetic islands which alter the topology of the field over substantial fractions of the plasma radius. For MST $q(0)$ usually has values slightly below 0.2 on 
axis and then decreases with radius passing through zero as $B_{\phi}$ passes through zero near the edge of the plasma. The innermost resonant mode is the $m=1, n=6$ although during a sawtooth crash we typically observe $q(0)$ rising above 0.2 bringing the $m=1, n=5$ briefly into resonance. These modes dominate higher $n, m=1$ modes which resonate farther out in the plasma where the plasma exhibits higher stabilizing shear. In addition to the core resonant $m=1$ modes, the MST plasma contains $m=0$ modes resonant at the reversal surface. These modes are dominated by the $m=0$, $n=1$ which may be responsible for the three-wave coupling of adjacent $m=1$ modes.

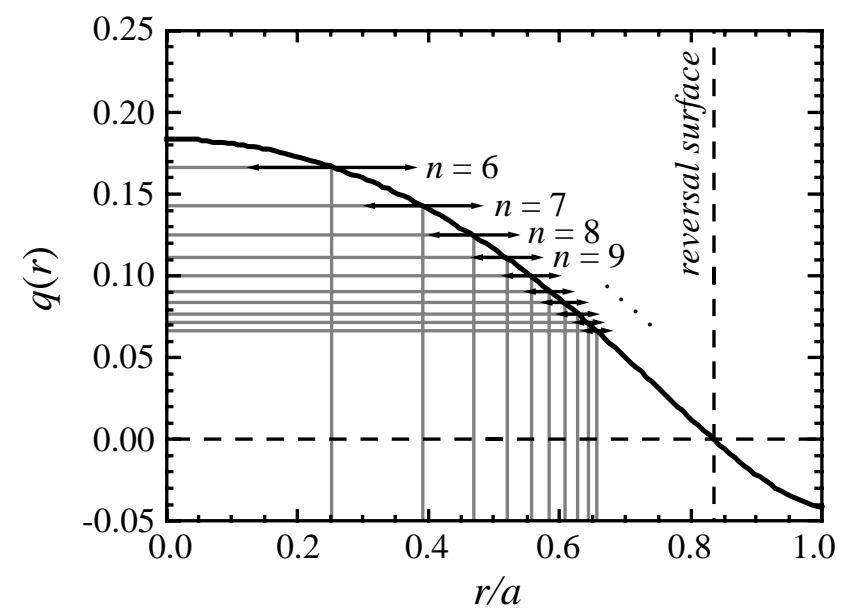

Fig. 3. A cylindrical model of the safety factor profile before a sawtooth crash for a typical, low current MST discharge. The horizontal arrows represent estimated island widths.

\section{MEASUREMENT OF $\langle\tilde{\mathbf{v}} \times \tilde{\mathbf{B}}\rangle$ IN MST}

A. The sawtooth cycle

MST is a large RFP with major radius $R=1.5 \mathrm{~m}$, minor radius $a=0.5 \mathrm{~m}$, and thick ( 5 $\mathrm{cm}$ ) aluminum conducting shell. To obtain the large ensemble datasets reported in this paper, MST was operated at a low plasma current of $200 \mathrm{kA}$ with discharge parameters $T_{e}=160 \mathrm{eV}$ and $n_{e}=$

$0.7 \times 10^{19} \mathrm{~m}^{-3}$. The evolution of the total toroidal flux over a shot (Fig. 4) is largely determined by the MST external circuit. The flux rises with the plasma current during the initial portion of the discharge and soon enters the "flat-top" region of the shot which may be maintained for tens of 
milliseconds before decaying. We restricted our analysis entirely to the flat-top region of the plasma during which a steady-state RFP equilibrium is most nearly achieved. During the flat-top region we see $\left\langle B_{\phi}\right\rangle_{V}$ decay and grow in a quasi-periodic sawtooth cycle. The signature event of this cycle is the sawtooth $\operatorname{crash}^{13,14}$ (also known as a "discrete dynamo event") during which $\left\langle B_{\phi}\right\rangle_{V}$ rapidly jumps by roughly $5-10 \%$ of its mean value (Fig. 4). The crashes are global events acting on all observable equilibrium quantities in a reproducible fashion. Fig. 5 shows the ensemble averaged sawtooth dynamics of two key equilibrium quantities. The reversal parameter, defined as the ratio of the edge toroidal field to the volume averaged toroidal field $\left(F \equiv B_{\phi}(a) /\left\langle B_{\phi}\right\rangle_{V}\right)$, becomes dramatically more negative during the crash before being pulled back close to its original value. The pinch parameter, defined as the ratio of the edge poloidal field to the volume averaged toroidal field $\left(\Theta \equiv B_{\theta}(a) /\left\langle B_{\phi}\right\rangle_{V}\right)$, drops due mostly to the rise of $\left\langle B_{\phi}\right\rangle_{V}$

in the denominator. Both effects reflect a flattening of the parallel current profile. The toroidal plasma current varies less than $1 \%$ over the sawtooth cycle. Although much of the detailed physics of the RFP sawtooth cycle is not understood, a useful phenomenological model describes the sawtooth as a discrete relaxation of a resistively peaked current profile. ${ }^{6}$

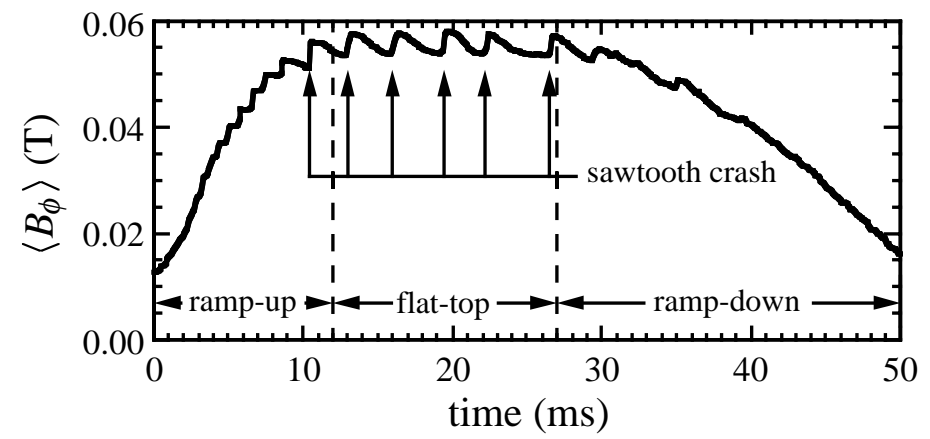

Fig. 4. The volume averaged toroidal field of a standard low current MST discharge. Note the well-defined sawteeth generating toroidal flux during the "flat-top" period. 


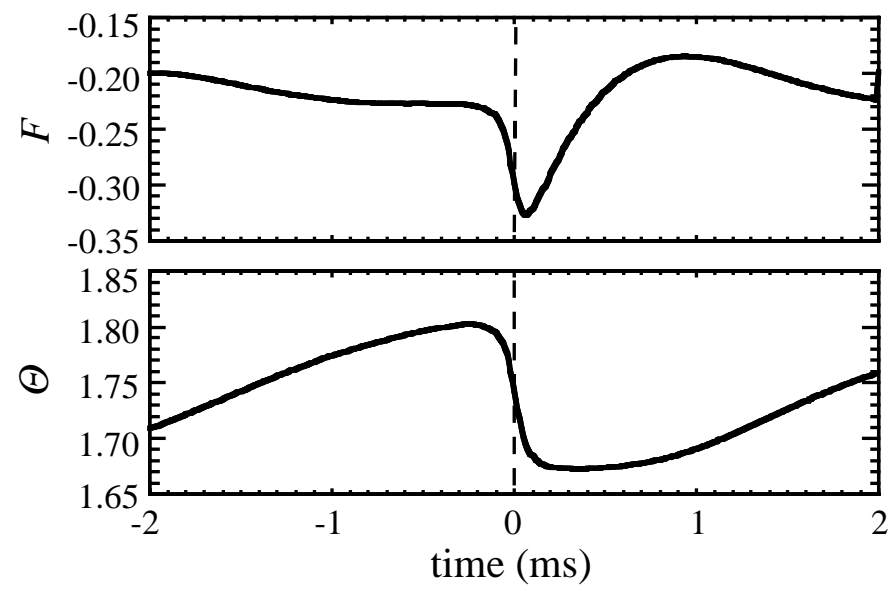

Fig. 5. Two key equilibrium quantities ensemble averaged over a sawtooth cycle: a) the reversal parameter $F$ and b) the pinch parameter $\Theta$.

B. Measurement of plasma flow velocity fluctuations

A central experimental innovation of this work is the passive spectroscopic measurement of core plasma velocity fluctuations. The key enabling component of this diagnostic achievement is the Ion Dynamics Spectrometer (IDS), which will only be briefly introduced here as this system and its calibration have been described in detail elsewhere. ${ }^{15,16,17}$. The IDS simultaneously records two chordal views of the plasma, each with 16 spectral wavelength channels (spreading out the Doppler-broadened spectrum over a large number of spectral channels decouples small fluctuations in the line centroid from those in the line width). The basic design of our spectrometer is that of an $f / 10$ Czerny-Turner monochromator with $1.0 \mathrm{~m}$ focal length. It is equipped with an $1180 \mathrm{~g} / \mathrm{mm}$ grating blazed at $1000 \mathrm{~nm}$; high dispersion is achieved by using this grating in fifth order when recording the $\mathrm{C}$ v $227.1 \mathrm{~nm}$ emission line. The most inexpensive way of optimizing system étendue was to use straight tall entrance slits (one slit above the optical midplane, the other below). The dominant aberration is then line curvature, which is caused by the variation in dispersion that occurs as a function of slit height. As long as the slit is fairly wide and not too tall, such line curvature can be compensated quite well by a simple tilt $\left(0.5^{\circ}\right.$ in our case $)$ of a straight slit. The dispersed spectra on the exit plane of the duo-spectrometer are coupled via fused silica fiber optic bundles to two arrays of 16 photomultiplier tubes each. All 32 photomultiplier tubes are 
read out and digitized at $1 \mathrm{MHz}$ in parallel. The result is a system which combines good étendue and resolution with the simplicity and flexibility of a fiber-optically-coupled single-grating spectrometer. Measurement precision is proportional to the impurity emission photon flux; in a typical MST discharge flow can be measured to a precision of $1 \mathrm{~km} / \mathrm{s}$ with $10 \mu$ s time resolution.

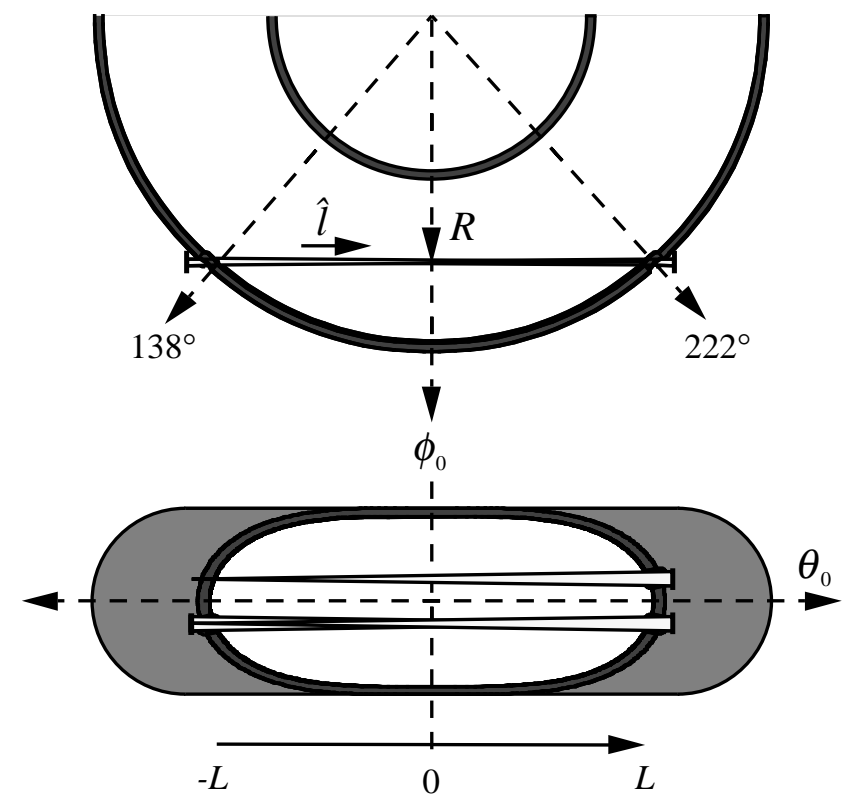

Fig. 6. The toroidal viewing chords, with both the opposing views and the views symmetrically placed above and below the mid-plane shown.

Several different viewing chords are used to measure toroidal, poloidal, and radial velocity and velocity flucutations in MST. Measurement of the toroidal plasma velocity is done using the lines of sight shown in Fig. 6. An average of the red- and blue-shifted emission lines from the opposing views provides as in situ calibration of the unshifted line position, making it simple to obtain an absolute measurement of the ion velocity. For measurement of a particular $m=1, n$, compoment of $\tilde{v}_{\phi}$, the views symmetrically placed above and below the mid-plane are employed to allow extraction of the local velocity fluctuation phase $\delta_{\phi}$ from the chord-averaged quantity: ${ }^{17,18}$

$$
\begin{aligned}
\left\langle\tilde{v}_{\phi}\right\rangle_{l} & =\frac{1}{2 L} \int_{-L}^{+L} I_{C V}\left|\tilde{v}_{\phi}\right| \exp \left[i\left(m \theta+n \phi+\omega t+\delta_{\phi}\right)\right](\hat{\phi} \cdot \hat{l}) d l \\
& =\frac{1}{a} \exp \left[i\left(m \theta_{T}+n \phi_{T}+\omega t\right)\right] \int_{r}^{a} I_{C V}\left|\tilde{v}_{\phi}\right| e^{i \delta_{\phi}} w_{\phi} d r
\end{aligned}
$$


where $I_{C V}$ is the impurity emission profile and $w_{\phi}$ is the appropriate geometric sensitivity function shown in Fig. 7. The instrument function quantifies the attenuation of the higher $k$ modes and illustrates the good sensitivity to the dominant $m=1, n=6$ mode in MST. Poloidal ion velocity fluctuations $\tilde{v}_{\theta}$ are measured using a boxport which allows collection at a variety of impact parameters. Radial velocity fluctuations $\tilde{v}_{r}$ are measured with a poloidal view with an impact parameter of zero. Analysis for the poloidal and radial viewing chords follows the pattern shown in Eq. (1), with a geometric sensitivity function which demonstrates good sensitivity to $m=1$ fluctuations of any toroidal mode number $n$.

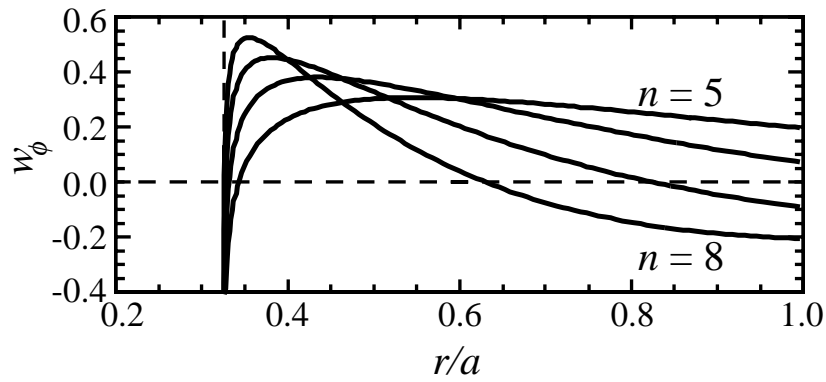

Fig. 7. The toroidal viewing chords geometric sensitivity functions $w_{\phi}$ for $m=1, n=5-8$.

The velocity fluctuations measured by the IDS may be expressed as the weighted sum of fluctuations with various wavenumbers, $\tilde{v}=\sum_{n} C_{n} \tilde{v}_{n}$, where $C_{n}$ depends on the convolution of the radial mode structure with the IDS instrument function and the $\mathrm{C} v$ emission profile. If we assume that all activity at the resonant wavenumbers in the plasma occurs with a coherent phase relation to the magnetic perturbations we may employ our knowledge of the magnetic perturbations to extract mode information from the velocity fluctuations. The rotation of the low $n$ magnetic modes converts their spatial structure in the plasma frame into a temporal variation in the lab frame. This allows the use of $\tilde{B}_{n}$ as a set of time dependent basis functions for the spatial Fourier analysis of $\tilde{v}$,

$$
\left\langle\tilde{v} \tilde{B}_{n}\right\rangle=\sum_{n^{\prime}} C_{n^{\prime}}\left\langle\tilde{v}_{n^{\prime}} \tilde{B}_{n}\right\rangle \approx C_{n}\left\langle\tilde{v}_{n} \tilde{B}_{n}\right\rangle
$$


By normalizing this product by the velocity and magnetic fluctuation power (i.e., calculating the coherence), we extract information about both $C_{n}$ and $\tilde{v}_{n}$.

Since the behavior of the majority ion species (or more specifically, the MHD single-fluid behavior of the plasma) is of greatest interest for our studies, the question naturally arises of how to relate IDS measurements of impurity ion dynamics to such quantities. Since the plasma flow velocity $\mathbf{v}=\left(\mathrm{m}_{i} \mathbf{v}_{i}+\mathrm{m}_{e} \mathbf{v}_{e}\right) /\left(\mathrm{m}_{i}+\mathrm{m}_{e}\right) \approx \mathbf{v}_{i}$, measurement of the ion flow should be sufficient for an MHD description of the plasma. The relationship of the impurity and majority flows has been explored in detail for tokamaks, ${ }^{19}$ but a similar analysis has not been done for the RFP, where magnetic field shear is substantial and the poloidal and toroidal magnetic fields are similar in magnitude. However, since the flow is expected to be dominated by $\mathbf{E} \times \mathbf{B}$ drifts and the collisional equilibration time between majority and impurity ions is approximately $50 \mu \mathrm{s}$, we expect that measurement of impurity flow quantitatively represents the behavior of the majority ions.

C. Measurement of the MHD dynamo

In summary, our procedure for measuring the MHD dynamo is the following:

- $\quad$ mode resolved measurement of $\tilde{B}$ with an array of 32 toroidally distributed magnetic pickup coils,

- $\quad$ spectroscopic measurement of $\tilde{v}$ from toroidal, poloidal, and radial views,

- $\quad$ approximate a flux surface average by an ensemble average over a large number of sawtooth cycles,

- $\quad$ correlate chord-averaged $\tilde{v}$ with dominant $\tilde{B}$ modes to resolve the toroidal mode number $n$ components of the dynamo product $\langle\tilde{\mathbf{v}} \times \tilde{\mathbf{B}}\rangle$.

The measured dynamo products are projected into the core using the known radial dependence of $\tilde{B}_{r}$ to obtain 


$$
\begin{aligned}
& \langle\tilde{\mathbf{v}} \times \tilde{\mathbf{B}}\rangle_{\theta}=\left\langle\tilde{v}_{\phi} \tilde{B}_{r}\right\rangle-\left\langle\tilde{v}_{r} \tilde{B}_{\phi}\right\rangle \\
& \langle\tilde{\mathbf{v}} \times \tilde{\mathbf{B}}\rangle_{\phi}=\left\langle\tilde{v}_{r} \tilde{B}_{\theta}\right\rangle-\left\langle\tilde{v}_{\theta} \tilde{B}_{r}\right\rangle
\end{aligned}
$$

The measured dynamo products are compared to estimates of $\eta J_{\|}-E_{\|}$from modeling to determine if Ohm's law, $E_{\|}+\langle\tilde{\mathbf{v}} \times \tilde{\mathbf{B}}\rangle_{\|}=\eta J_{\|}$, is balanced.

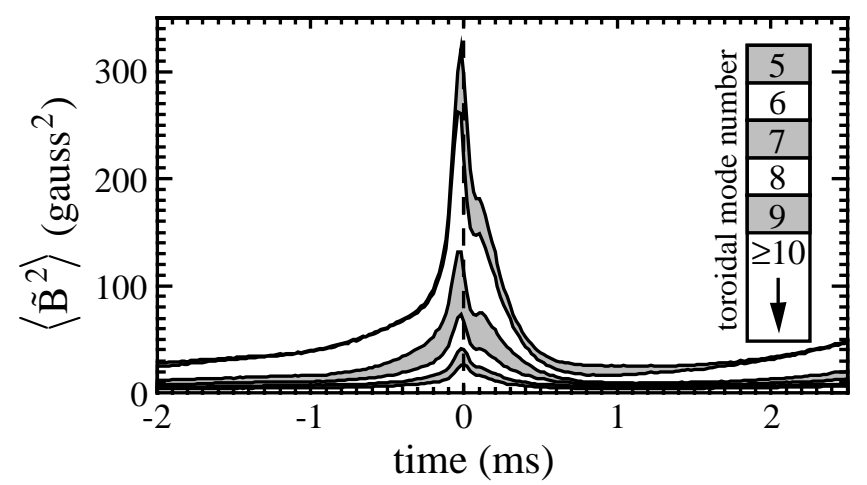

Fig. 8. $\tilde{B}_{\theta, n}^{2}(t)$ ensembled over a sawtooth window. The total magnetic fluctuation power is traced out by the top curve. The light and dark bands indicate the distribution of power among the dominant toroidal modes as indicated by the key.

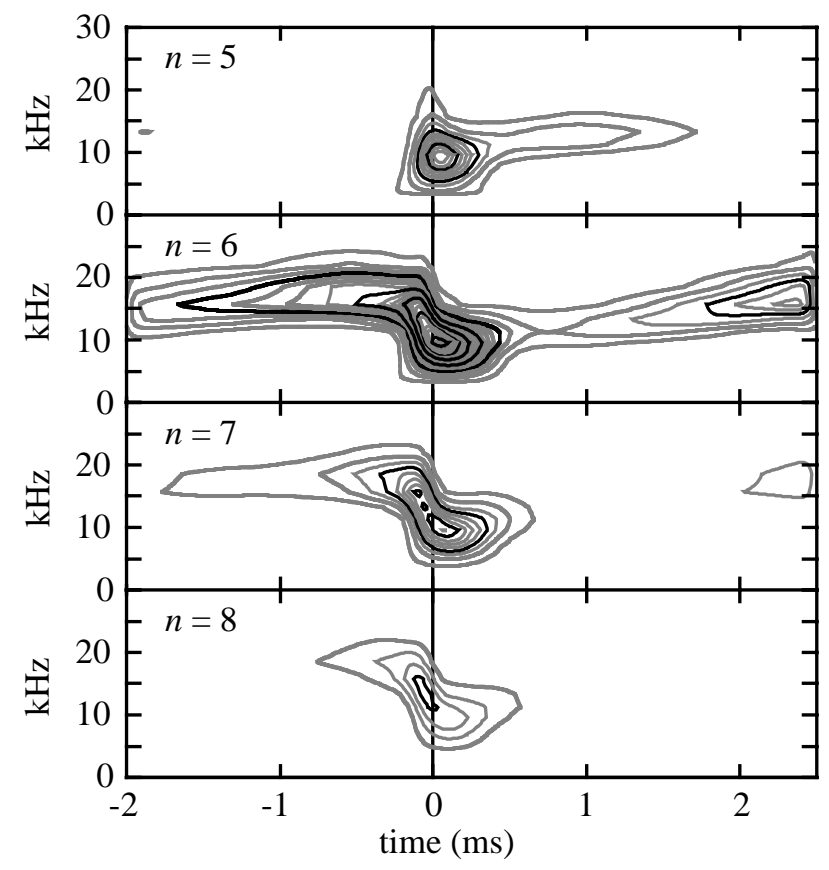

Fig. 9. Wavelet spectra of the magnetic fluctuation power density of dominant $m=1$ modes over a sawtooth cycle. 
As described above in Sec. II, the magnetic fluctuation spectrum in MST is dominated by core resonant, $m=1$, low $n$ resistive tearing modes. The fluctuation power peaks dramatically at the sawtooth crash, as illustrated in Fig. 8 by measurements of mode resolved $\tilde{B}_{\theta}$ ensembled over a sawtooth window. The fluctuations are nearly stationary in the plasma frame, but rotate at 8-20 $\mathrm{kHz}$ in the lab frame, as illustrated in the wavelet power spectra in Fig. 9. The modes decelerate sharply at the sawtooth crash, accelerating more slowly following the crash. ${ }^{20}$ Also at the crash the fluctuation spectrum broadens and the $n=5$ mode comes into resonance.
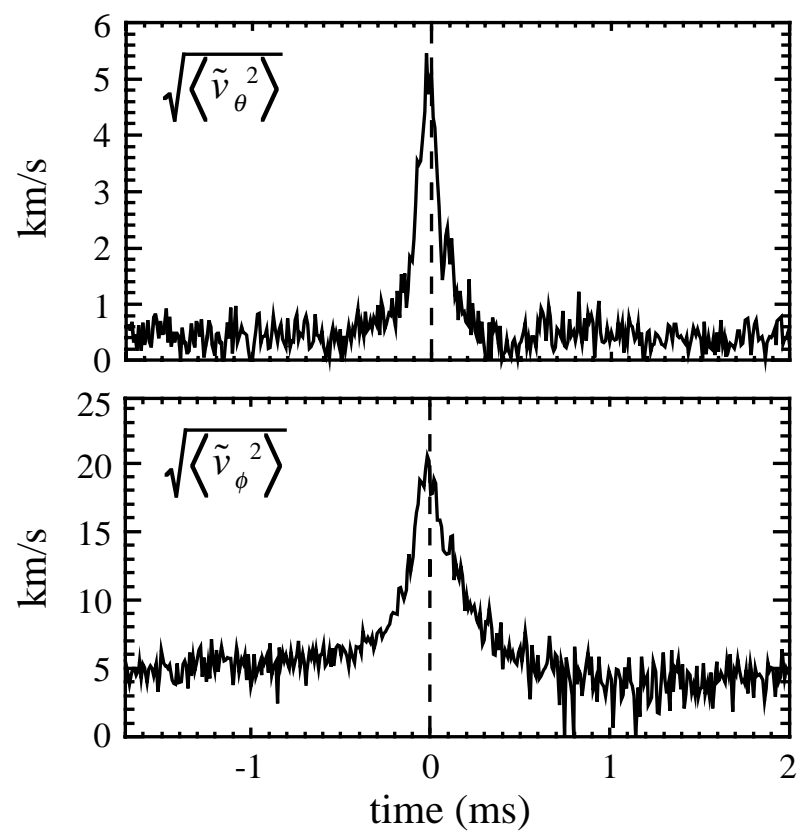

Fig. 10. The rms amplitudes of the poloidal and toroidal velocity fluctuations over a sawtooth cycle.

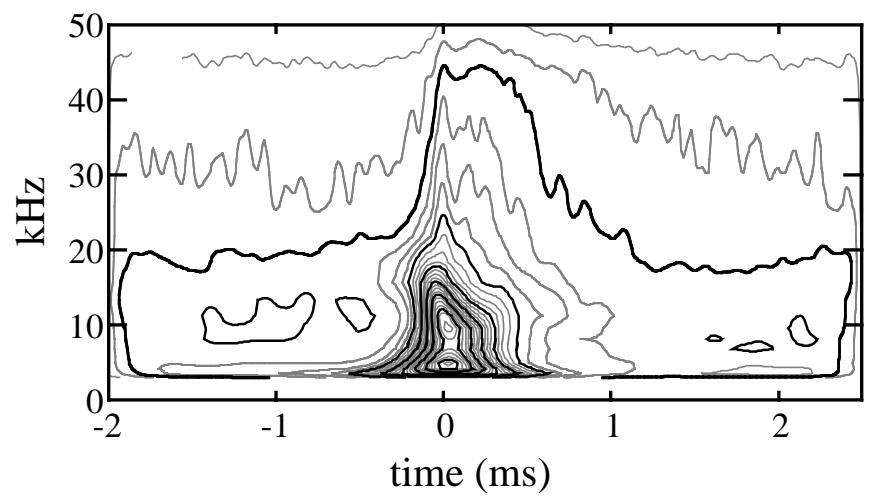

Fig. 11. Wavelet spectra of the toroidal velocity fluctuation power density over a sawtooth cycle. 

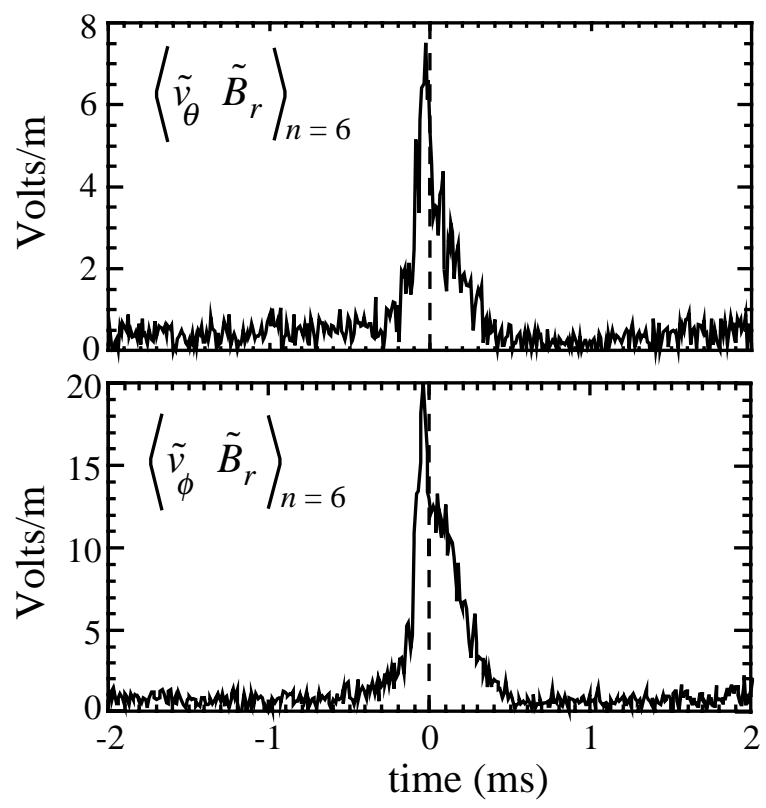

Fig. 12. Two $n=6$ components of the dynamo, both of which suppress parallel current in the core of MST. Note the dramatic peaking of the MHD dynamo at the sawtooth crash.

As measured by the IDS, the velocity fluctuation power spectrum is not only attenuated by the instrument function as described above, but also contains noise power due to photon counting statistics and the sudden change in plasma flow that occurs at a sawtooth crash. The photon noise is white, i.e., it has a flat power spectrum that is quantified and subtracted off. The power from the secular evolution of the plasma flow peaks at low frequency with a high frequency tail due to rapid changes at the sawtooth crash. This tail is fit by an exponential decay and also subtracted from the power spectra. The results are plotted in Fig. 10, the rms velocity fluctuation amplitude over a sawtooth window, and Fig. 11, the wavelet spectra of total fluctuation power. As with the magnetic fluctuations, the fluctuation power dramatically peaks at the sawtooth crash, and the fluctuation frequency downshifts as the plasma decelerates. As might be expected from the behavior of the magnetic and velocity fluctuations individually, the MHD dynamo product $\langle\tilde{\mathbf{v}} \times \breve{\mathbf{B}}\rangle$, is large at the sawtooth crash and small otherwise. Fig. 12 shows two components of the dynamo, both of which suppress parallel current in the core of MST. The velocity and magnetic fluctuations reach peak coherence at the sawtooth crash and are nearly in phase, maximizing the dynamo product. The dominant uncertainty in the measured dynamo field is not 
statistical, but instead is the systematic uncertainties in the estimate of the chordal attenuation of $\tilde{v}$ and the extrapolation of edge $\tilde{B}$ to fluctuation amplitude in the core. The exact size of this uncertainty is difficult to calculate without prior knowledge of the fluctuation eigenfunctions, but we estimate it to be in the range of $30-50 \%$.

Surprisingly, the measured radial velocity fluctuations do not appear to play a role in the MHD dynamo in the core of MST. The radial velocity fluctuation power is smaller, but roughly comparable to that in the toroidal and poloidal components, yet the coherence of $\tilde{v}_{r}$ with the dominant $m=1$ magnetic fluctuations barely exceeds the coherence baseline. Thus the contribution of $\left\langle\tilde{v}_{r} \tilde{B}_{\theta}\right\rangle$ and $\left\langle\tilde{v}_{r} \tilde{B}_{\phi}\right\rangle$ to the measured dynamo fields is negligible compared to the $\left\langle\tilde{v}_{\phi} \tilde{B}_{r}\right\rangle$ and $\left\langle\tilde{v}_{\theta} \tilde{B}_{r}\right\rangle$ terms contained in Eqs. (3a) and (3b). This result seems to indicate that dynamo field generation by a large-scale $m=1$ circulating flow pattern is not likely. There are at least two other possibilities. First, the radial viewing chord, while it has good geometric sensitivity to a large-scale $m=1$ velocity fluctuation, has no sensitivity to an $m=0$ flow fluctuation. There is good evidence that a substantial $m=0, n=1$ magnetic perturbation, resonant on the $q=0$ surface, exists in MST. ${ }^{21}$ This mode may be an important component of the MHD dynamo. $^{22}$ Second, the $m=1$ flow fluctuation may have finer scale structure than suggested by 3 dimensional resistive MHD simulation. In particular, if the $m=1$ radial flow fluctuations important in the MHD dynamo reverse sign when crossing a rational surface (such as the $q=1 / 6$ surface in the core of MST), then the IDS radial viewing chord would average such fluctuations to zero. However, the puzzle remains as to why the measured $\tilde{v}_{r}$, which are not insignificant, are not correlated with $\tilde{B}$.

There is additional evidence that the velocity fluctuation eigenfunctions may be somewhat localized to the rational surfaces, with widths similar to the island widths (Fig. 3). This is in contrast to magnetic fluctuation eigenfunctions, which both simulation and experiment confirm are 
global, with substantial amplitude across the minor radius. ${ }^{18}$ Evidence for the localization of the velocity fluctuations comes from the poloidal viewing chord measurements of the dynamo product. These measurements have been taken at several $r / a$ impact parameters. At a specific impact parameter intersecting a $q=1 / n$ resonant surface, the measured $\tilde{v}_{\theta}$ fluctuations exhibit significant coherence predominantly with the $m=1$ magnetic fluctuations resonant at that surface. Thus the mode-resolved $\left\langle\tilde{v}_{\theta} \tilde{B}_{r}\right\rangle_{n}$ peaks at the location of the resonant surface in the plasma. Dividing the mode-resolved dynamo product by the $\tilde{B}$ of that mode gives an estimate of the mode-resolved velocity fluctuation amplitude, plotted versus impact parameter in Fig. 13. This plot suggests that the velocity fluctuation eigenfunction for a particular $n$-mode peaks near the $1 / n$ resonant surface.

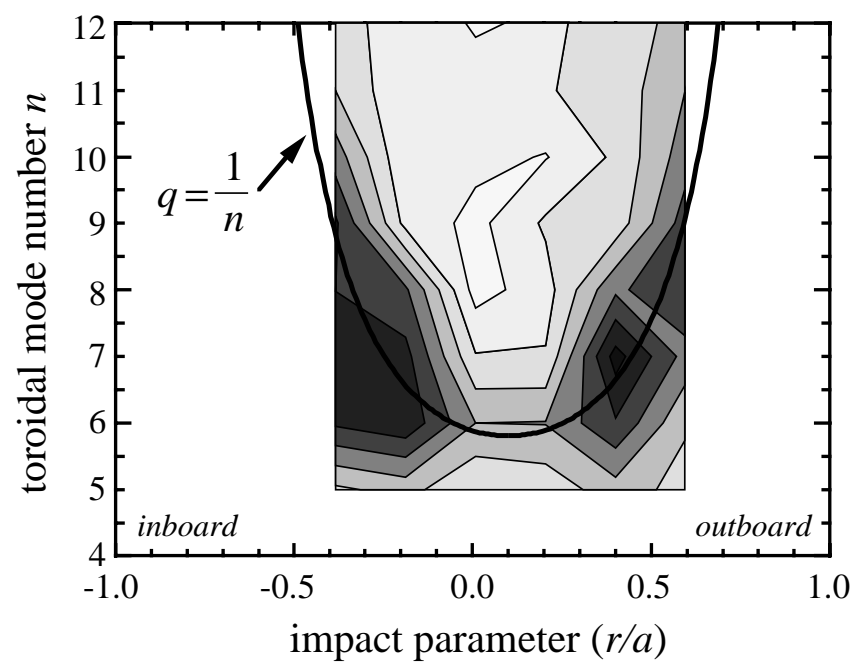

Fig. 13. Amplitude contours of mode-resolved $\left(\sqrt{\tilde{v}_{\theta}^{2}}\right)_{n}$, estimated by dividing the mode-resolved dynamo product $\left\langle\tilde{v}_{\theta} \tilde{B}\right\rangle_{n}$ by $\tilde{B}$ for that mode, measured at various poloidal viewing chord impact parameters. The velocity fluctuation amplitude for a particular $n$-mode peaks approximately at the impact parameter where that mode is expected to be resonant (estimated from the $q$-profile), indicating that the velocity fluctuation eigenfunction for a particular $n$-mode peaks near the $1 / n$ resonant surface in the plasma.

Since it appears that radial velocity fluctuations do not play a major role in the MHD dynamo product in the core of MST, comparison to Ohm's law reduces to comparison of the 
toroidal and poloidal components of $\eta J_{\|}-E_{\|}$to $\left\langle\tilde{v}_{\theta} \tilde{B}_{r}\right\rangle$ and $\left\langle\tilde{v}_{\phi} \tilde{B}_{r}\right\rangle$, respectively. Modeling of a parallel Ohm's law as a function of minor radius in MST is complicated by two factors. First, there is limited experimental measurement of the actual current density profile in MST, so it has been estimated from equilibrium modeling of edge and global measurements. Second, during the sawtooth crash, rapid relaxation of the current profile induces a transient electric field of tens of $\mathrm{V} / \mathrm{m}$ which greatly exceeds both the externally applied field and the electric field necessary to drive current in the plasma. Thus, during the crash, the dynamo term implied by this modeling must act to suppress parallel current in the core with a field of 20-40 V/m and drive current in the edge with a field of 5-15 V/m (Fig. 14). During the rest of the sawtooth cycle, the inductance of the plasma opposes the resistive decay of the equilibrium, thus Ohm's law is approximately balanced without a contribution from the RFP dynamo (Fig. 14). Comparison to the actual measured dynamo products at the sawtooth crash is shown in Fig. 15, where the toroidal and poloidal components of Fig. 14 have been separately plotted. Agreement is relatively good, implying that in MST the simple parallel Ohm's law $E_{\|}+\langle\tilde{\mathbf{v}} \times \tilde{\mathbf{B}}\rangle_{\|}=\eta J_{\|}$, is approximately balanced by the MHD dynamo.

Of course, this initial measurement has limited spatial localization and there are large uncertainties arising from extrapolation of the amplitude of the dynamo product into the core, so much refinement is still needed.

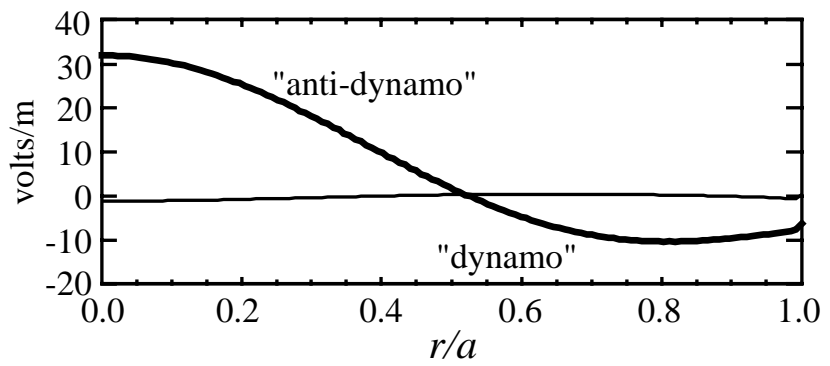

Fig. 14. From Ohm's law modeling, the electric field $\eta J_{\|}-E_{\|}$that must be supplied by the RFP dynamo during (heavy line) and away (light line) from the sawtooth crash. "Anti-dynamo" refers to suppression of $J_{\|}$in the core, and "dynamo" refers to driving of $J_{\|}$in the edge. 

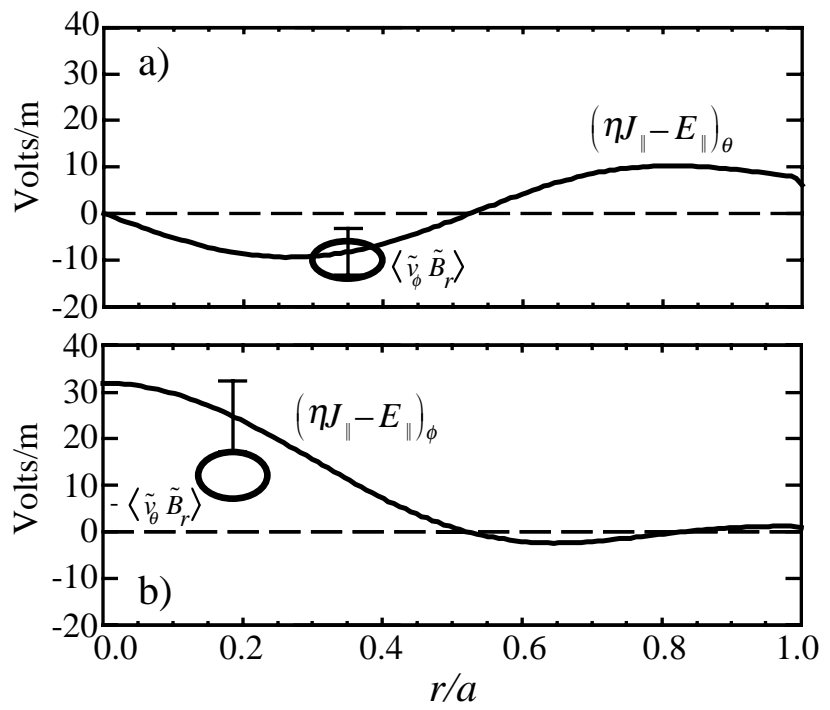

Fig. 15. The a) poloidal and b) toroidal components of $\eta J_{\|}-E_{\|}$estimated from modeling, compared to two experimental measurements of the MHD dynamo at the sawtooth crash. The uncertainties in $\eta J_{\|}-E_{\|}$are represented by error bars; the uncertainties in spatial localization and amplitude of the dynamo measurements are represented by the heavy oval datapoints.

D. Edge measurement of the MHD dynamo

Further evidence for the important role of the MHD dynamo in balancing Ohm's law in MST is supplied by a novel optical probe capable of providing spatially resolved velocity

fluctuation measurements in the edge of MST. Since this probe is described in detail elsewhere, ${ }^{23}$ only a brief overview will be given here. As with the IDS measurements of the core plasma, the principle is again Doppler spectroscopy, but of the bright He II $468.6 \mathrm{~nm}$ emission line in the edge of MST. Optical radiation is collected by two fused silica fiber optic bundles with perpendicular viewlines. Spatial resolution of about $5 \mathrm{~cm}$ is achieved by terminating each view with an optical dump. The collected light is transported by the fiber bundles to the IDS. Two components of the velocity are measured simultaneously — the radial along the insertion of the probe and a perpendicular component which can be varied by simply rotating the probe by $90^{\circ}$. The accuracy of the velocity measurements is better than $1 \mathrm{~km} / \mathrm{s}$. The probe is armored by a boron nitride enclosure and has been inserted to $r / a=0.8$ in the edge of MST. 


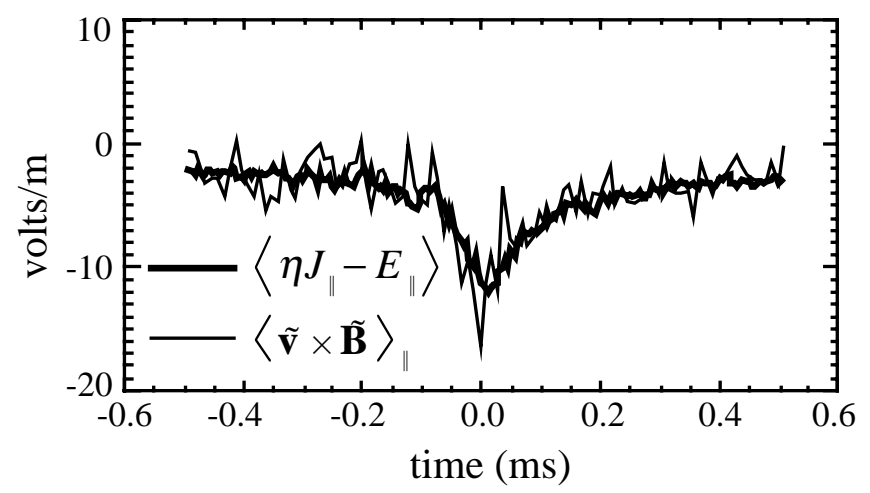

Fig. 16. Comparison of the ensemble averaged MHD dynamo product measured with the optical probe at $r / a=0.9$ to parallel Ohm's law during the sawtooth crash.

Comparison of the MHD dynamo product measured at $r / a=0.9$ to parallel Ohm's law is shown in Fig. 16. The agreement is striking, and confirms earlier measurements with a complex Langmuir probe. ${ }^{7}$ However, the same measurement made deeper into the plasma, around the $q=$ 0 surface at $r / a \approx 0.80$, do not exhibit the same agreement with simple Ohm's law modeling. The cause of this disagreement is currently unknown; it may be an instrumental artifact resulting from perturbation of the plasma as the probe is inserted more deeply, or it may be that terms other than $\langle\tilde{\mathbf{v}} \times \tilde{\mathbf{B}}\rangle$ are required to balance Ohm's law.

\section{UNDERSTANDING AND CONTROLLING THE DYNAMO}

In MST, the MHD dynamo appears to be the primary mechanism by which the RFP magnetic field configuration is maintained against resistive decay. The dynamo term, $\langle\tilde{\mathbf{v}} \times \tilde{\mathbf{B}}\rangle$, is large during the sawtooth crash and overcomes the transient inductive electric field to suppress parallel current in the core and drive parallel current in the edge. Specifically, we have measured the plasma flow velocity fluctuations $\tilde{\mathbf{v}}$ with a fast, high-resolution spectrometer, the magnetic field fluctuations $\tilde{\mathbf{B}}$ with an edge coil array, and correlated the two quantities to show that $\langle\tilde{\mathbf{v}} \times \tilde{\mathbf{B}}\rangle_{\|}$

approximately balances parallel Ohm's law, $E_{\|}+\langle\tilde{\mathbf{v}} \times \tilde{\mathbf{B}}\rangle_{\|}=\eta J_{\|}$. Contributions to the dynamo

product appear to be dominated by the $\left\langle\tilde{v}_{\theta} \tilde{B}_{r}\right\rangle$ and $\left\langle\tilde{v}_{\phi} \tilde{B}_{r}\right\rangle$ components; the measured radial 
velocity fluctuations are not coherent with the dominant $m=1$ magnetic fluctuations. In addition, the velocity fluctuation eigenfunctions appear to be more localized to the rational surfaces than the magnetic fluctuation eigenfunctions, which have significant amplitude across the entire minor radius.

Thus, we have made significant progress in understanding the fluctuations in MST that relax the plasma to a minimum energy state while conserving magnetic helicity. But, these same fluctuations cause substantial particle and energy transport in the RFP. ${ }^{24,25}$ Quoting Sykes and Wesson from their paper on the original RFP simulation: "It appears therefore that a reverse field configuration can be maintained indefinitely, the key question then being whether the loss of confinement arising from the level of instability required to maintain the reversal is acceptable or not." The answer to this question appears to be negative, since all standard RFP's exhibit poorer confinement than competing concepts such as the tokamak. Therefore, a key to the future of the RFP as a magnetic confinement fusion reactor will be controlling or even eliminating the fluctuations that drive the dynamo. Progress towards this goal has been made in transient experiments in which the emf normally supplied by the dynamo is externally applied, ${ }^{26}$ and plans have been made to extend this to steady-state current drive techniques. A rich area of future application of the measurement techniques described in this paper will be to examine the response of RFP plasma fluctuations to such current profile control. Also now being applied to the RFP are charge-exchange recombination spectroscopy ${ }^{27}$ and Rutherford scattering ${ }^{28}$ of a diagnostic neutral beam to measure the detailed structure and character of the flow fluctuation patterns. These experimental techniques will overcome the lack of spatial localization that limits the present measurements. The knowledge thus gained will have input not only to fusion research, but to many questions of basic plasma physics.

\section{ACKNOWLEDGEMENT}

We gratefully acknowledge the contribution of many members of the MST Group to the success of this study. This work was supported by the U. S. Department of Energy. 


\section{REFERENCES}

1R. N. Dexter, D. W. Kerst, T. W. Lovell, S. C. Prager, and J. C. Sprott, Fusion Technol. 19, 131 (1991).

2J. B. Taylor, Phys. Rev. Lett. 33, 1139 (1974); Rev. Mod. Phys. 58, 741 (1986).

3H. Ji, S. C. Prager, and J. S. Sarff, Phys. Rev. Lett. 74, 2945 (1995).

4D. C. Robinson and R. E. King, Proceedings of the Third International Conference on Plasma Physics and Controlled Nuclear Fusion Research, Novosibirsk, 1968, (IAEA, Vienna, 1969), Vol. I, p. 263.

5A. Sykes and J. A. Wesson, Proceedings of the Eighth European Conference on Controlled Fusion and Plasma Physics, Prague, 1977, (Czechoslovak Academy of Sciences, Prague, 1977), Vol. I, p. 80.

6S. Ortolani and D. D. Schnack, Magnetohydrodynamics of Plasma Relaxation (World Scientific, Singapore, 1993).

7H. Ji, S. C. Prager, A. F. Almagri, J. S. Sarff, Y. Yagi, Y. Hirano, K. Hattori, and H. Toyama, Phys. Plasmas 3, 1935 (1996).

8A. Jacobson and R. Moses, Phys. Rev. Lett. 52, 2041 (1984).

9G. Giruzzi and E. Martines, Phys. Plasmas 1, 2653 (1994); E. Martines and F. Vallone, Phys. Rev. E 56, 957 (1997).

10P. W. Terry and P. H. Diamond, Phys. Fluids B 2, 1128 (1990).

11M. R. Stoneking, Ph.D. thesis, University of Wisconsin-Madison, 1993.

12F. Krause and K.-H. Rädler, Mean-Field Magnetohydrodynamics and Dynamo Theory (Pergamon Press, 1980); H. K. Moffatt, Magnetic Field Generation in Electrically Conducting Fluids (Cambridge University Press, 1978).

13S. Hokin, A. Almagri, S. Assadi, et al., Phys. Fluids B 3, 2241 (1991).

14J. A. Beckstead, Ph.D. thesis, University of Wisconsin-Madison, 1990.

15D. J. Den Hartog and R. J. Fonck, Rev. Sci. Instrum. 65, 3238 (1994).

16J. T. Chapman and D. J. Den Hartog, Rev. Sci. Instrum. 68, 285 (1997).

17D. J. Den Hartog, A. F. Almagri, J. T. Chapman, G. Fiksel, C. C. Hegna, and S. C. Prager, Fizika Plazmy 24, 169 (1998) [Plasma Phys. Reports 24, 148 (1998)].

18J. T. Chapman, Ph.D. thesis, University of Wisconsin-Madison, 1998.

19Y. B. Kim, P. H. Diamond, and R. J. Groebner, Phys. Fluids B 3, 2050 (1991). 
20D. J. Den Hartog, A. F. Almagri, J. T. Chapman, R. J. Fonck, C. C. Hegna, H. Ji, S. C Prager, and J. S. Sarff, Phys. Plasmas 2, 2281 (1995).

21D. Craig, A. F. Almagri, J. K. Anderson, et al., Phys. Rev. Lett. 79, 1865 (1997).

22Gunnar Hedin, Plasma Phys. Control. Fusion 40, 1529 (1998).

23G. Fiksel, D. J. Den Hartog, and P. W. Fontana, Rev. Sci. Instrum. 69, 2024 (1998).

24G. Fiksel, S. C. Prager, W. Shen, and M. Stoneking, Phys. Rev. Lett. 72, 1028 (1994).

25M. R. Stoneking, S. A. Hokin, S. C. Prager, G. Fiksel, H. Ji, and D. J. Den Hartog, Phys. Rev. Lett. 73, 549 (1994).

26M. R. Stoneking, N. E. Lanier, S. C. Prager, J. S. Sarff, and D. Sinitsyn , Phys. Plasmas 4, 1632 (1997).

27H. T. Evensen, R. Durst, R. J. Fonck, and S. F. Paul, Rev. Sci. Instrum. 66, 845 (1995); H. T. Evensen, R. J. Fonck, S. F. Paul, G. Rewoldt, S. D. Scott, W. M. Tang, and M. C. Zarnstorff, Nucl. Fusion 38, 237 (1998).

28D. J. Den Hartog, G. Fiksel, V. Davydenko, A. Ivanov, and V. Mishagin, “A Diagnostic Neutral Beam System for the MST Reversed-Field Pinch: CHERS and Rutherford Scattering," abstract to be published in Rev. Sci. Instrum. 